\title{
ESPLENECTOMIA PARCIAL PARA TRATAR HEMANGIOMA ESPLÊNICO
}

\author{
PARTIAL SPLENECTOMY FOR TREATMENT OF SPLENIC HEMANGIOMA
}

\author{
Andy Petroianu, TCBC-MG'; Carlos Teixeira Brandt, TCB -MG²; Lúcio Flávio de Alencar³
}

\begin{abstract}
RESUMO: Apesar de a esplenectomia ser eficaz na abordagem terapêutica de pacientes com hemangioma esplênico, esse procedimento é acompanhado de elevada morbidade e até mortalidade, principalmente devido à sepse, quando realizado em crianças e adolescentes com sistema imunitário ainda imaturo. Para prevenir os efeitos adversos da asplenia, propõe-se neste artigo a esplenectomia parcial, com a retirada apenas da região do hemangioma, mantendo o restante do baço e preservando suas importantes funções (Rev. Col. Bras. Cir. 2008; 35(2): 132-135).
\end{abstract}

Descritores: Hemangioma; Esplenectomia; Baço; Preservação biológica; Literatura de revisão.

\section{INTRODUÇÃO}

Com o objetivo de preservar as funções esplênicas, desde 1984 realizamos a esplenectomia subtotal combinada com derivação esplenorrenal ou desconexão portavarizes, para tratar hipertensão porta esquistossomática em 122 doentes 1,2. Esplenectomia subtotal foi também realizada em 59 pacientes com trauma esplênico grave ${ }^{3,4}$, nove pacientes com hepatoesplenomegalia mielóide ${ }^{5}$, seis pacientes com doença de Gaucher ${ }^{6}$, quatro pacientes com retardo de desenvolvimento somático e sexual associado a esplenomegalia ${ }^{7}$, um paciente com doença de Hodgkin, um paciente com leucemia linfocítica crônica e um paciente com cistoadenoma corpocaudal de pâncreas ${ }^{8,9,10}$.

Mesmo sendo raro, o hemangioma é a neoplasia mais comum do baço ${ }^{11}$. Os relatos de estudos em necropsias, apontam para incidências do hemangioma esplênico que variam entre $0,03 \%$ e $14 \%{ }^{11,12,13}$. Na maior parte dos casos, ele é assintomático e seu diagnóstico se faz por acaso em um exame de imagem do abdome, por outros motivos ${ }^{13,14}$. Suas dimensões são geralmente menores de quatro centímetros, porém o hemangioma pode crescer e, nesse caso, acompanha-se de desconforto no hipocôndrio esquerdo ${ }^{11,12}$. Por ser uma afecção congênita, as manifestações surgem freqüentemente na infância e adolescência, mas podem também iniciar na fase adulta ${ }^{12}$.

O risco mais grave dos hemangiomas maiores é a sua ruptura com conseqüente hemorragia grave ${ }^{12,15}$. Calcula-se que cerca de um quarto dos hemangiomas grandes rompem, portanto a indicação do tratamento operatório torna-se inquestionável ${ }^{12,15}$. Apesar de existirem alguns relatos de embolização dos vasos que nutrem o hemangioma ${ }^{15,16,17}$ e de terapia antiangiogênica ${ }^{18}$, o tratamento proposto na literatura é a esplenectomia total por laparotomia ou laparoscopia $11,12,14,19,20,21,22$.

Apesar de a esplenectomia total resolver definitivamente os problemas decorrentes do hemangioma, ela cria uma doença muito grave, que é a asplenia. Atualmente, não há mais dúvida da grande importância do baço, principalmente como órgão de defesa contra infecções e responsável por diversas funções metabólicas ${ }^{23}$. Quadros sépticos graves são muito mais comuns em esplenectomizados do que na população em geral, a ponto de a sepse estar envolvida no óbito em três quartos dos asplênicos, contra menos de um quarto dos que possuem baço ${ }^{24,25}$. Alterações no metabolismo das bilirrubinas, de diversos aminoácidos e dos lipídios também são encontradas após a retirada completa do baço ${ }^{23}$.

Para evitar as complicações decorrentes da esplenectomia e a morte precoce, são cada vez mais reduzidas as indicações cirúrgicas no baço, tanto em trauma quanto em doenças hematológicas, metabólicas ou oncológicas, além da hipertensão porta. Se a esplenectomia for inevitável, cabe ressaltar a opção pelas operações conservadoras, com especial destaque para a esplenectomia parcial, a esplenectomia subtotal e os auto-implantes esplênicos no omento maior, quando houver a retirada completa do baço ${ }^{1-10,26,27,28}$.

O objetivo deste trabalho é apresentar a esplenectomia parcial como uma boa opção terapêutica para hemangioma do baço. Este trabalho teve por base um paciente do sexo masculino, feodérmico, com 13 anos de idade procurou o Serviço de Cirurgia Pediátrica da Faculdade de Medicina da Universidade Federal de Pernambuco com dor testicular intensa e persistente. Ao exame físico foi diagnosticada varicocele apenas esquerda. Para investigar a causa dessa

1. Professor Titular do Departamento de Cirurgia da Faculdade d Medicina, UFMG; Docente Livre em Técnica Operatória e Cirurgia Experimental da Escola Paulista de Medicina, UNIFESP; Docente Livre em Gastroenterologia Cirúrgica da Faculdade de Medicina de Ribeirão Preto, USP; Doutor em Fisiologia e Farmacologia - Instituto de Ciências Biológicas, UFMG, Pesquisador IA do CNPq.

2. Professor Titular de Cirurgia Pediátrica da Universidade Federal de Pernambuco.

3. Cirurgião Pediátrico; Mestre em Saúde da Criança e do Adolescente - Universidade Federal de Pernambuco.

Recebido em 20/08/2007

Aceito para publicação em 30/10/2007

Conflito de interesses: nenhum

Fonte de financiamento: nenhuma

Trabalho realizado na Universidade Federal de Pernambuco e Hospital HOPE de Recife, Pernambuco. 
anomalia vascular, solicitou-se exame ultra-sonográfico de abdome, que revelou um tumor arredondado de pólo inferior do baço, que poderia estar comprimindo a entrada da veia testicular esquerda na veia renal. A ressonância magnética confirmou o tumor esplênico, com característica de hemangioma.

\section{TÉCNICA}

A indicação operatória fundamentou-se na presença de um grande hemangioma sintomático. Por ser uma afecção benigna e localizada em apenas um segmento do baço, optouse pela esplenectomia parcial com a retirada do segmento esplênico doente.

O acesso operatório foi por uma incisão subcostal esquerda de dez centímetros. Inicialmente, ligou-se a artéria esplênica no espaço retrogástrico, através de abertura do omento maior e ligamento gastrocólico.

Em seguida, foram seccionados os ligamentos frenocólico, esplenorrenal e suspensor do baço, para mobilizar todo o baço para o campo operatório. A metade inferior do baço foi exteriorizada e os vasos polares para essa região foram ligados e seccionados. Delineou-se o limite entre o terço distal do baço, azulado pela isquemia, e o restante do órgão, que permaneceu róseo.

O baço foi seccionado em cunha, com a retirada da parte isquemiada, que continha o hemangioma e foi removida com margem livre. A hemostasia dos vasos parenquimatosos foi com sutura, utilizando fio de categute cromado 3-0 agulhado. Esse mesmo fio foi utilizado para suturar a cápsula esplênica sobre a área cruenta do baço. O remanescente esplênico foi colocado em seu leito. Por último, realizou-se a sutura da parede abdominal por planos anatômicos.

O procedimento operatório foi conduzido quase sem sangramento e não houve dificuldade técnica.

O paciente recebeu dieta no dia seguinte à operação e alta hospitalar, em bom estado geral, no segundo dia pósoperatório.

O exame anatomopatológico da peça retirada confirmou a presença de um grande hemangioma cavernoso de baço.

No acompanhamento superior a oito meses, não houve intercorrência alguma e o paciente retornou às suas atividades normais desde a segunda semana após a operação. A dor testicular desapareceu e o aspecto da varicocele melhorou. Os exames hematológicos e de imagem do baço permaneceram normais durante todo o período pós-operatório.

\section{DISCUSSÃO}

Os avanços na pesquisas sobre a importância funcional baço e sua ampla divulgação são responsáveis pela grande redução na indicação das operações esplênicas no último decênio. Apesar de ainda existirem profissionais que, por limitações técnicas ou desconhecimento, ainda realizam esplenectomias totais rotineiramente, já há a tendência de muitos cirurgiões em optarem pelas operações esplênicas conservadoras, quando não houver indicação estabelecida de retirada completa do baço, como no câncer, no hiperesplenismo ou na púrpura trombocitopênica refratários a tratamento medicamentoso ${ }^{1-10,26,27,28}$.

As operações sobre o baço têm por objetivo interromper o sangramento, no trauma, reduzir o desconforto de baços gigantes, na doença de Gaucher, na esplenomegalia mielóide e em leucemias, reduzir o fluxo sangüíneo porta, nas afecções com hipertensão porta, além de remover neoplasias benignas desse órgão, nos cistos, leiomiomas e hemangiomas. Em todos esses casos não há necessidade de remover todo o baço e tornar o paciente asplênico, com todas as complicações desse estado, incluindo a morte precoce.

As operações conservadoras incluem desde a sutura e desvascularização segmentar do baço, em traumas menores, até as esplenectomias parciais e as subtotais. Eventualmente, a preservação de parte do baço eutópico pode ser difícil ou o cirurgião não estar habituado com as técnicas para esse procedimento. Nesses casos, pode-se realizar a esplenectomia total seguida de auto-implantes esplênicos no omento maior, que é uma das operações abdominais mais fáceis e rápidas 29,30,31,32,33,34 . Todos esses procedimentos são eficazes e previnem a asplenia.

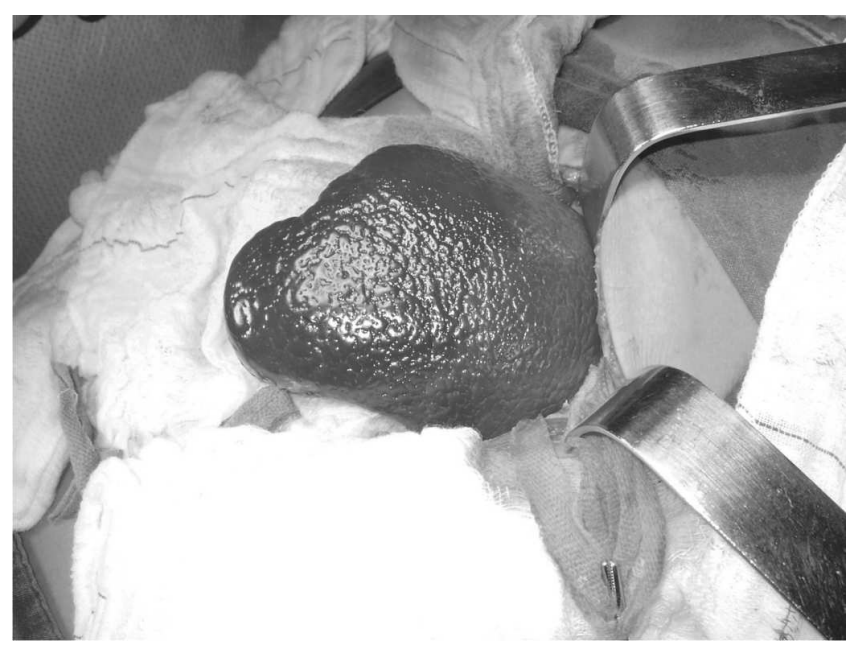

Figura 1 - a - Operação para retirada de hemangioma localizado no pólo inferior do baço. Observar o hemangioma de cor mais escura.

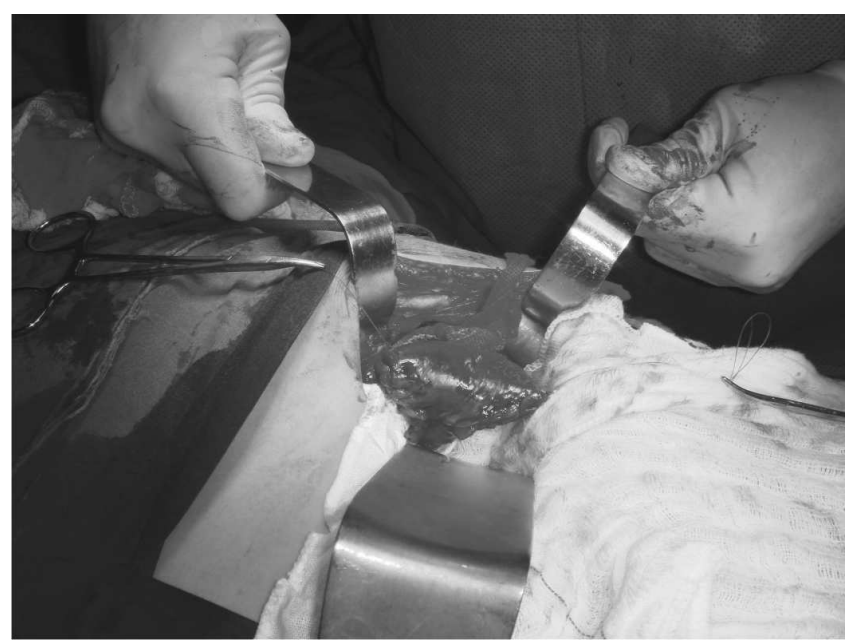

Figura 1 - b-Remanescente esplênico após a remoção do hemangioma e sutura da cápsula esplênica. 
Esta Nota Técnica destaca pela primeira vez na literatura nacional e segunda vez na mundial ${ }^{22}$ a esplenectomia parcial para tratar hemangioma esplênico. Após a mobilização completa do baço para o campo operatório e a ligadura dos vasos que irrigam e drenam a região esplênica em que se encontra o hemangioma, há um perfeito domínio do baço e sua secção parcial, para a remoção do tumor, torna-se muito fácil. A literatura menciona muitos métodos para hemostasia do parênquima ${ }^{26,27,28}$, porém apenas a sutura dos vasos, seguida pela síntese da cápsula é o mais simples.

Em nossa experiência superior a 250 esplenectomias subtotais, não houve complicação provocada pelo remanescente esplênico $^{1-10}$. Nos casos em que não pudemos realizar a esplenectomia parcial ou subtotal, optamos pelo implante de 20 fragmentos de tecido esplênico no omento maior, para pre- servar tecido esplênico suficiente, com drenagem para a veia porta, o que garante sua perfeita função, confirmada por diversos estudos ${ }^{32,33,34,35,36}$.

Apesar do bom resultado obtido com o presente caso, é necessário o estudo de uma casuística maior e por um tempo mais prolongado para garantir a eficácia da esplenectomia parcial para tratar hemangioma esplênico. Um outro avanço que deverá ser adotado em situações parecidas é a esplenectomia parcial por via laparoscópica ${ }^{20,21,26,37}$, procedimento que já realizamos previamente, com sucesso, em dois doentes.

O bom resultado obtido e a facilidade técnica na realização da esplenectomia parcial para tratar hemangioma esplênico mostra ser essa uma boa alternativa terapêutica, que retira completamente a doença, mantendo o restante do órgão, sem os riscos que acompanham a asplenia.

\begin{abstract}
Background: Although splenectomy is helpful in the management of splenic hemangioma, this procedure may be accompanied by a greater morbidity and even mortality, mainly caused by sepsis, when this operation is performed in children and teenagers, due to their immune deficiency. In order to avoid the adverse effects of the asplenism, this paper proposes partial splenectomy to treat splenic hemangioma.
\end{abstract}

Key words: Hemangioma; Splenectomy; Spleen; Preservation, biological; Review literature.

\section{REFERÊNCIAS}

1. Petroianu A. Treatment of portal hypertension by subtotal splenectomy and central splenorenal shunt. Postgrad Med J. 1988; 64(747):38-41.

2. Petroianu A. Subtotal splenectomy and portal-variceal disconnection in the treatment of portal hypertension. Can $\mathbf{J}$ Surg. 1993; 36(3):251-4.

3. Resende V, Petroianu A. Subtotal splenectomy for treatment of severe splenic injuries. J Trauma. 1998; 44(5):933-5.

4. Resende V, Petroianu A. Functions of the splenic remnant after subtotal splenectomy for treatment of severe splenic injuries. Am J Surg. 2003; 185(4):311-5.

5. Petroianu A. Subtotal splenectomy for treatment of patients with myelofibrosis and myeloid metaplasia. Int Surg. 1996; 81(2):177-9.

6. Petroianu A. Subtotal splenectomy in Gaucher's disease. Eur J Surg. 1996; 162(6):511-3.

7. Petroianu A. Subtotal splenectomy for treatment of retarded growth and sexual development associated with splenomegaly. Minerva Chir. 2003; 58(3):413-4.

8. Petroianu A. Treatment of cystadenoma of the pancreatic tail by distal pancreatectomy and subtotal splenectomy. Dig Surg. 1995; 12(4):259-61.

9. Petroianu A, Silva RG, Simal CJ, Carvalho DG, Silva RA. Late postoperative follow-up of patients submitted to subtotal splenectomy. Am Surg. 1997; 63(8):735-40.

10. Petroianu A, Resende V, Silva RG. Late follow-up of patients submitted to subtotal splenectomy. Int J Surg. 2006; 4(3):152-8. Epub 2006 Feb 20.

11. Willcox TM, Speer RW, Schlinkert RT, Sarr MG. Hemangioma of the spleen: presentation, diagnosis, and management. $\mathrm{J}$ Gastrointest Surg. 2000; 4(6): 611-3.
12. Husni EA. The clinical course of splenic hemangioma with emphasis on spontaneous rupture. Arch Surg. 1961; 83:681-8

13. Phillpott J, Ali SA, Briscoe EG, Cesani F. Three-phase Tc-99m labeled RBC scintigraphy of a splenic hemangioma. Clin Nucl Med. 1997; 22(3):158-60.

14. Kato M, Lubitz C, Finley D, Chadburn A, Fahey TJ. Splenic cord capillary hemangioma and anemia: resolution after splenectomy. Am J Hematol. 2006; 81(7):538-42.

15. Norris PM, Hughes SC, Strachan CJ. Spontaneous rupture of a benign cavernous haemangioma of the spleen following thrombolysis. Eur J Vasc Endovasc Surg. 2003; 25(5):476-7.

16. Brandt CT, Rothbarth LJ, Kumpe D, Karrer FM, Lilly JR. Splenic embolization in children: long-term efficacy. J Pediatr Surg. 1989; 24(7):642-4; discussion 644-5.

17. Smith HE, Biffl WL, Majercik SD, Jednacz J, Lambiase R, Cioffi WG. Splenic artery embolization: have we gone too far? J Trauma. 2006; 61(3):541-6.

18. Islam S, Newman EA, Strouse PJ, Geiger JD. Antiangiogenic therapy for a large splenic hemangioma. Pediatr Surg Int. 2005; 21(12):1007-10. Epub 2005 Oct 8.

19. Tsugawa K, Hashizume M, Migou S, Kawanaka H, Sugimachi K, Irie H, Maeda T, Akaboshi K. Laparoscopic splenectomy for an inflammatory pseudotumor of the spleen: operative technique and case report. Hepatogastroenterology. 1998; 45(23):188791.

20. Uchida H, Ohta M, Shibata K, Endo Y, Iwaki K, Tominaga M, Ishio T, Kitano S. Laparoscopic splenectomy in patients with inflammatory pseudotumor of the spleen: report of 2 cases and review of the literature. Surg Laparosc Endosc Percutan Tech. 2006; 16(3):182-6.

21. Yano H, Imasato M, Monden T, Okamoto S. Hand-assisted laparoscopic splenectomy for splenic vascular tumors; report of two cases. Surg Laparosc Endosc Percutan Tech. 2003; 13(4):286-9 
22. Pampin C, Devillers A, Treguier C, Fremond B, Moisan A, Goasguen J, Le Gall E. Intratumoral consumption of indium-111labeled platelets in a child with splenic hemangioma and thrombocytopenia. J Pediatr Hematol Oncol. 2000; 22(3):256-8.

23. Cooper MJ, Williamson RCN. Splenectomy: indications, hazards and alternatives. Br J Surg. 1984; 71(3):173-80.

24. Offenbartl K. Postsplenectomy sepsis. Bull Lund Univ. 1985; 56:4-65.

25. Pimpl W, Dapunt O, Kaindl H, Thalhamer J. Incidence of septic and thromboembolic-related deaths after splenectomy in adults. Br J Surg. 1989; 76(5):517-21.

26. Chazalette JP, Feigelson J, Louis D. Partial splenectomy-worth the risk. Arch Dis Child. 2003; 88(7):649.

27. Upadhyaya P. Conservative management of splenic trauma: history and current trends. Pediatr Surg Int. 2003; 19(9-10):61727. Epub 2003 Nov 12.

28. Itamoto T, Fukuda S, Tashiro H, Hideki O, Asahara T. Radiofrequency-assisted partial splenectomy with a new and simple device. Am J Surg. 2006; 192(2):252-4.

29. Leemans R, Manson W, Snijder JA, Smit JW, Klasen HJ, The TH, Timens W. Immune response capacity after human splenic autotransplantation: restoration of response to individual pneumococcal vaccine subtypes. Ann Surg. 1999; 229(2):279-85.

30. Iinuma H, Okinaga K, Sato S, Tomioka M, Matsumoto K. Optimal site and amount of splenic tissue for autotransplantation. J Surg Res. 1992; 53(2):109-16.

31. Lanng Nielsen J, Saksø P, Hanberg Sørensen F, Hvid Hansen H. Demonstration of splenic functions following splenectomy and autologous spleen implantation. Acta Chir Scand. 1984; 150(6):469-73.
32. Petroianu A, Petroianu A, Vidigal FM, Costa VC, Oliveira SC. Splenic autotransplantation in Gaucher's disease. Dig Surg. 2000; 17(2):181-3.

33. Petroianu A, Petroianu LP. Splenic autotransplantation for treatment of portal hypertension. Can J Surg. 2005; 48(5):3826.

34. Resende V, Petroianu A, Junior WC. Autotransplantation for treatment of severe splenic lesions. Emerg Radiol. 2002; 9(4):20812. Epub 2002 Aug 9.

35. Petroianu A, Simal CJ, Barbosa AJ. Assessment of phagocytic function in remnants of subtotal splenectomy and in autologous spleen implantation. Med Sci Res. 1993; 21(19):715-7.

36. Marques RG, Petroianu A, Coelho JM, Portela MC. Regeneration of splenic autotransplants. Ann Hematol. 2002; 81(11):622-6. Epub 2002 Nov 9.

37. Petroianu A, Berindoague Neto R. Laparoscopic subtotal splenectomy. Minerva Chir. 2004; 59(5):501-5.

Como citar este artigo:

Andy Petroianu A, Brandt CT, Alencar LF. Esplenectomia parcial para tratar hemangioma esplênico. Rev Col Bras Cir. 2007; 34(4).

Disponível em URL: www.scielo.br/rcbc

Endereço para correspondência:

Prof. Andy Petroianu

Avenida Afonso Pena, 1626 - apto. 1901

30130-005 - Belo Horizonte- MG

Telefone/Fax : 31-3274-7744 e 8884-9192

E-mail:petroian@medicina.ufmg.br 